\title{
Experiencia de innovación docente universitaria en la enseñanza de la hidrología
}

\author{
CARMEN ZARZUELO ROMERO \\ Universidad de Sevilla \\ Escuela de Ingeniería \\ Departamento de Ingeniería \\ Aeroespacial y Mecánica de Fluidos. \\ czarzuelo@us.es \\ ORCID: http://orcid.org/0000-0003-4551-5510 \\ D.O.I.: http://dx.doi.org/10.12795/JDU.2018.i01.72 \\ Pp.: 1290-1306
}

\section{Resumen}

Se presenta el ciclo de mejora desarrollado en la asignatura Hidrología superficial, en el bloque de Pérdidas de Precipitación y Transformación Lluvia Caudal. El objetivo de las sesiones era que el alumnado fuese capaz de entender que no todo lo que llueve forma escorrentía, para lo que tienen que conocer los usos y tipos de suelo y las condiciones de humedad/sequía previas. La mejora pretendía que el alumnado tomara un mayor protagonismo dejando al docente en un segundo plano. Las tres primeras sesiones resultaron caóticas, el alumnado se vio perdido y no fue capaz de continuar sin directrices. Sin embargo, en la última sesión se empezó a vislumbrar en algunos/ as de ellos/as que son capaces de desarrollar las ideas sin necesidad de una guía. Quizás el desarrollo innovador de las sesiones deba ser 
implementado desde el inicio de las asignaturas para que se habitúen al método de trabajo, que inicialmente les resulta complejo.

Palabras clave: Hidrología superficial y subterránea, Grado en Ingeniería Civil, Docencia Universitaria, Experimentación Docente Universitaria.

\section{Breve descripción del contexto}

Las sesiones del ciclo de mejora están enmarcadas en la asignatura "Hidrología superficial y subterránea", asignatura obligatoria de la intensificación de hidrología de 4음 curso del Grado de Ingeniería Civil. Se trata de una asignatura de 6 créditos. Constituye el punto de partida para el desarrollo del resto de las asignaturas de la intensificación ya que el fin de la asignatura es calcular el caudal de agua, variable de partida para el diseño de obras hidráulicas o marítimas.

En ella hay matriculados 23 alumnos/as y asisten normalmente los 23 aunque en la clase de los Viernes (15:0016:50) suele haber bajas (5-6 alumnos/as). Hay un alumno Erasmus (procedente de Alemania), 3 alumnos que van a curso por año y, el resto, son de años anteriores aunque cursando por primera vez la asignatura. Este ciclo de mejora ha sido desarrollado durante 4 clases de 1 hora $50 \mathrm{mi}$ nutos de duración, por tanto durante un total de 7 horas y 20 minutos.

La asignatura se desarrolla con el objetivo de profundizar sobre el conocimiento y la compresión de la Hidrología, ya que previamente el alumnado ha sido iniciado con conceptos básicos en la asignatura de 20 Curso "Hidráulica e hidrología", así como en el manejo de herramientas informáticas que les permitan desarrollar todo lo que hasta ahora había sido calculado a mano. Se pretende por tanto que el alumnado sea capaz de enfrentarse a un estudio hidrológico, determinando que parámetros necesita conocer y los pasos a seguir hasta conseguir el fin impuesto. Por otro lado, deben ser capaces al final de la asignatura de interpretar los resultados para identificar los posibles fallos ocasionados en el desarrollo del estudio hidrológico y suplirlos. 
El ciclo de mejora se va a centrar en el bloque de las pérdidas de precipitación y en la transformación lluvia caudal (Tema 5 y 6 del programa de la asignatura), dos temas que suelen ser delicados por no conocer los conceptos básicos.

\section{Diseño previo del ciclo de mejora}

Los contenidos teóricos fueron desarrollados en base a la estructura expuesta por diferentes profesores de otras Universidades (Nanía, 2006; Sánchez San Román, 2017), así como por manuales de hidrología aplicada (Chow, 1994). En base a ello se diseñó el mapa de contenidos que es mostrado a continuación en la Figura 1.

El mapa de contenidos parte de una pregunta inicial que permite desarrollar cada una de las partes de ambos temas. La pregunta es: ¿Todo lo que llueve forma escorrentía? La respuesta permite introducir los conceptos y aspectos teóricos del tema a desarrollar, así como de la metodología usada para su cálculo. Pero la separación de la lluvia de la lluvia efectiva (la que forma escorrentía) no siempre se usa, depende del método para transformar lluvia-caudal que se quiera usar. Esto último permite introducir el segundo tema y explicar los diferentes métodos usados y los parámetros que se necesitan conocer para cada uno de ellos. En todo el mapa de contenidos no sólo tienen que quedarse con los aspectos teóricos, sino que también tienen que profundizar en la representación y la interpretación de los resultados, y como estos pueden verse modificados según cambien las condiciones de partida.

A continuación se diferencian algunos de los contenidos según sea conceptuales (marcados en naranja en el mapa), procedimentales (en azul) o actitudinales (en verde):

Jornadas de Formación e Innovación Docente del Profesorado I № 1 (2018) Esta obra se distribuye con la licencia Creative Commons 


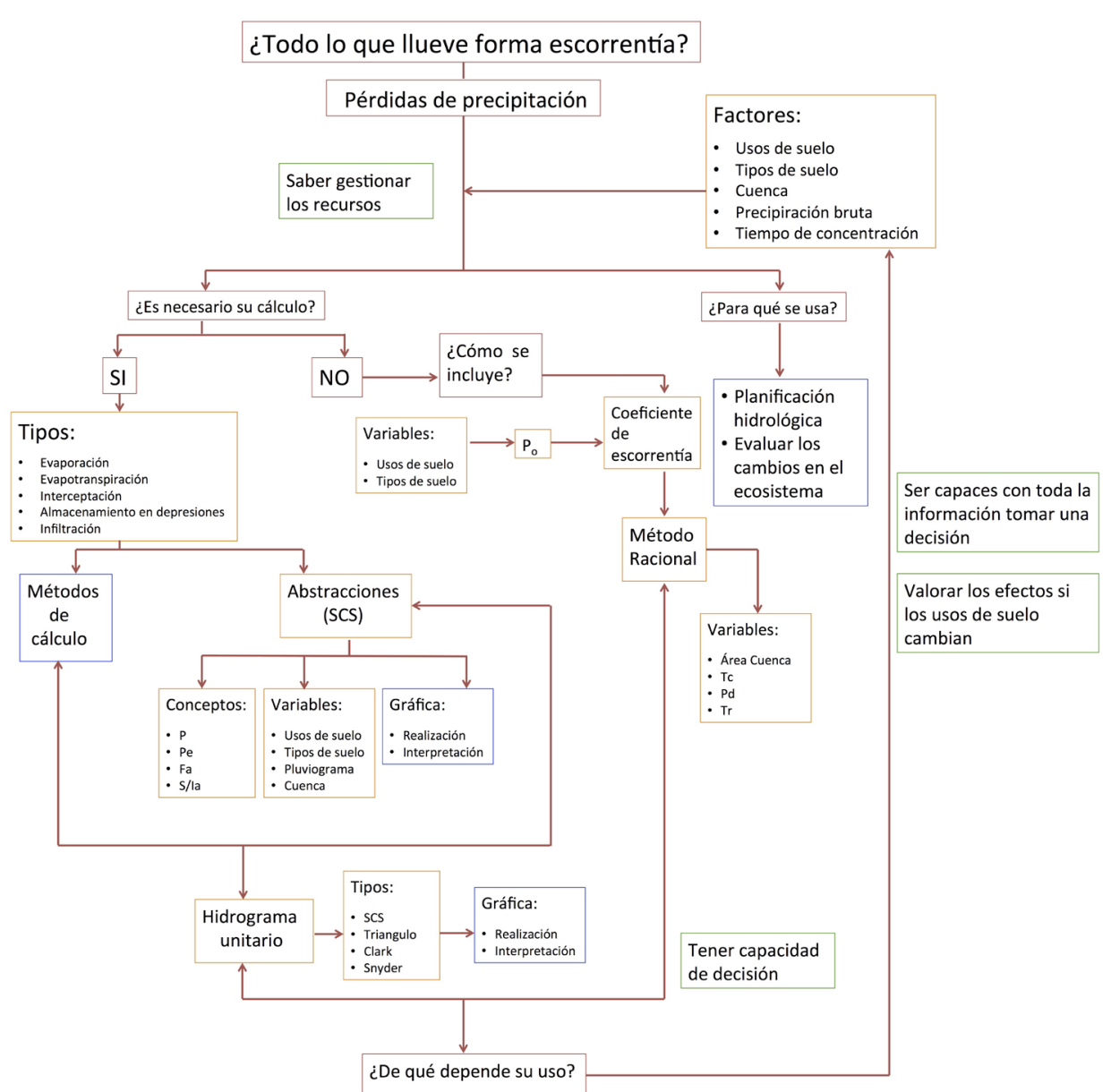

Figura 1.- Mapa de Contenido de los temas: (1) Pérdidas de Precipitación y (2) Transformación Lluvia-Caudal

- Contenidos conceptuales: Conocer y definir las características de la cuenca, usos, tipos de suelo y tiempo de concentración, que son clave para el cálculo de las pérdidas.

- Contenidos procedimentales: Seleccionar los métodos de cálculo más acordes a las características de la zona de estudio; ser capaces de realizar la planificación hidrológica; saber gestionar los recursos existentes en la cuenca para una buena gestión y 
planificación y, por último, evaluar los cambios del ecosistema.

- Contenidos actitudinales: Interés por realizar una buena gestión y planificación; desarrollo de autonomía en la toma de decisiones e integración de la información modificada en la nueva toma de decisión.

El modelo metodológico que hemos considerado posible en este momento es el que se muestra en la Figura 2. El modelo ha sido diseñado de esta manera para adecuarse en primer lugar a los contenidos y competencias que se quiere que adquieran los alumnos/as. Se persigue que entiendan los conceptos básicos, sepan aplicarlos y sepan tomar decisiones en base a eso. En la primera parte del modelo se busca el replanteamiento de las ideas concebidas por ellos/as mediante el trabajo con casos reales, donde tengan que buscar solución usando los conceptos adquiridos durante los años de la carrera. Esto sirve como punto de partida para el desarrollo de los temas.

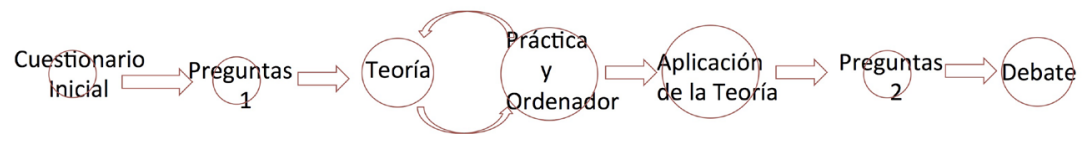

Figura 2.- Modelo metodológico implementado durante el ciclo de mejora.

La primera actividad sería el Cuestionario Inicial, donde se plantean preguntas abiertas sobre conceptos básicos y claves del tema a desarrollar. La siguiente actividad sería la denominada como Preguntas 1, donde se plantean una o dos preguntas acerca de una imagen o un vídeo que aborden el por qué se estudia ese tema dentro de la hidrología. Las respuestas del alumnado serían recogidas por el docente en la pizarra y servirían para delimitar la respuesta final. A continuación se iría intercalando el desarrollo de la teoría con la puesta en práctica mediante problemas sencillos; la parte de teoría durará 2-3 minutos, con un problema corto de otros 3-4 minutos; se repetiría este proceso todas las veces que fueran necesarias. 
La siguiente actividad sería la más importante, es donde el alumnado debe de poner en práctica todo lo anterior en un caso real que se irá desarrollando durante todo el cuatrimestre; la aplicación será tanto con herramientas informáticas como con calculadora, lápiz y papel. Por último, quedarían dos actividades que irían conjuntamente, la primera sería lo que hemos llamado Preguntas 2, donde se plantean preguntas que los estudiantes deben contestar de manera individual para después ser debatidas en clase. Primero debatirán entre ellos sobre la posible respuesta y luego el docente les ayudará a definir la mejor respuesta; posteriormente pueden reescribir lo que han escrito y, finalmente, serán recogidas las respuestas para ser corregidas por el docente.

A continuación se muestra la secuencia de actividades desarrolladas en cada sesión:

Tabla 1. Secuencia de actividades diseñada

\begin{tabular}{|l|l|l|}
\hline \multicolumn{3}{|l|}{ Sesión 1: ¿Todo lo que llueve forma escorrentía? } \\
\hline $\begin{array}{l}\text { Fase del Modelo } \\
\text { Metodológico }\end{array}$ & Descripción de la actividad & Tiempo \\
\hline Cuestionario inicial & $\begin{array}{l}\text { Se reparte el cuestionario inicial se les } \\
\text { deja tiempo para resolver las cuestiones } \\
\text { y se recoge. }\end{array}$ & $15^{\prime}$ \\
\hline Pregunta 1 & $\begin{array}{l}\text { Se les plantean preguntas acerca de una } \\
\text { imagen. Las preguntas están centradas } \\
\text { en contestar conceptos claves de la uni- } \\
\text { dad a desarrollar. Son preguntas abier- } \\
\text { tas, donde pueden ser contestadas desde } \\
\text { diferentes puntos de vista. Las ideas de } \\
\text { cada alumno/a son recogidas en la piza- } \\
\text { rra para finalmente entre todos/as fijar la } \\
\text { respuesta más acertada. }\end{array}$ & 7 \\
\hline
\end{tabular}

Jornadas de Formación e Innovación Docente del Profesorado | № 1 (2018) Esta obra se distribuye con la licencia Creative Commons 


\begin{tabular}{|l|l|}
\hline Teoría & $\begin{array}{l}\text { Realización del esquema de todo estu- } \\
\text { dio hidrológico definiendo con detalle la } \\
\text { parte correspondiente a lo dado en clase } \\
\text { y las demás parte con pinceladas. El es- } \\
\text { quema es realizado entre todos y escrito } \\
\text { por el docente en la pizarra. }\end{array}$ \\
\hline
\end{tabular}

Sesión 2: Maneras de cálculos de la precipitación efectiva

\begin{tabular}{|c|c|c|}
\hline $\begin{array}{l}\text { Fase del Modelo } \\
\text { Metodológico }\end{array}$ & Descripción de la actividad & Tiempo \\
\hline Pregunta 1 & $\begin{array}{l}\text { Es expuesto una imagen con la represen- } \\
\text { tación de todas las variables en las que } \\
\text { se dividen la precipitación con el fin de } \\
\text { poder introducir el tema. }\end{array}$ & $10^{\prime}$ \\
\hline Teoría-Práctica & $\begin{array}{l}\text { Se van introduciendo conceptos teóricos } \\
\text { durante } 2-3 \text { minutos y los siguientes 3-4 } \\
\text { minutos se pone en práctica lo anterior. Y } \\
\text { es repetido hasta el final de la actividad. } \\
\text { Introducir los modelos y los métodos de } \\
\text { cálculo. }\end{array}$ & $35^{\prime}$ \\
\hline $\begin{array}{l}\text { Aplicación a la } \\
\text { realidad }\end{array}$ & $\begin{array}{l}\text { Los procesos y conceptos aprendidos du- } \\
\text { rante la anterior actividad son puestos en } \\
\text { práctica en el caso real que tiene que ser } \\
\text { entregado al final del curso. }\end{array}$ & $40^{\prime}$ \\
\hline Preguntas 2 & $\begin{array}{l}\text { Se plantean dos preguntas sobre el tema } \\
\text { (se les recuerda que pueden ser pregun- } \\
\text { tas de examen) para que contesten sobre } \\
\text { la marcha de manera individual. }\end{array}$ & $10^{\prime}$ \\
\hline Debate & $\begin{array}{l}\text { Las preguntas anteriores son debati- } \\
\text { das de manera colectiva sin la ayuda del } \\
\text { docente para que posteriormente vaya } \\
\text { guiando la respuesta correcta. }\end{array}$ & $15^{\prime}$ \\
\hline \multicolumn{3}{|c|}{ Sesión 3: Variables y parámetros influyentes } \\
\hline $\begin{array}{l}\text { Fase del Modelo } \\
\text { Metodológico }\end{array}$ & Descripción de la actividad & Tiempo \\
\hline Pregunta 1 & $\begin{array}{l}\text { Se realiza la recapitulación entre todos/ } \\
\text { as donde se irán anotando todo lo dicho } \\
\text { por parte del alumnado tanto acertado } \\
\text { como erróneo hasta definir el correcto } \\
\text { esquema. }\end{array}$ & $15^{\prime}$ \\
\hline
\end{tabular}

Jornadas de Formación e Innovación Docente del Profesorado | № 1 (2018) Esta obra se distribuye con la licencia Creative Commons 


\begin{tabular}{|c|c|c|}
\hline Teoría-Práctica & $\begin{array}{l}\text { Se van introduciendo conceptos teóricos } \\
\text { durante 2-3 minutos y los siguientes 3-4 } \\
\text { minutos se pone en práctica lo anterior. } \\
\text { Y es repetido hasta el final de la activi- } \\
\text { dad. Se introducen las variables y pará- } \\
\text { metros necesarios para el cálculo donde } \\
\text { pueden ser encontradas y como acudir a } \\
\text { ellas. También se les introduce los posi- } \\
\text { bles cambios de las mismas asi como sus } \\
\text { posibles modificaciones en los cálculos. }\end{array}$ & $30^{\prime}$ \\
\hline $\begin{array}{l}\text { Aplicación a la } \\
\text { realidad }\end{array}$ & $\begin{array}{l}\text { Los procesos y conceptos aprendidos du- } \\
\text { rante la anterior actividad son puestos en } \\
\text { práctica en el caso real que tiene que ser } \\
\text { entregado al final del curso. Se hace uso } \\
\text { de la herramienta ArcGis. }\end{array}$ & $40^{\prime}$ \\
\hline Preguntas 2 & $\begin{array}{l}\text { Se plantean dos preguntas sobre el tema } \\
\text { (se les recuerda que pueden ser pregun- } \\
\text { tas de examen) para que contesten sobre } \\
\text { la marcha de manera individual. }\end{array}$ & $10^{\prime}$ \\
\hline Debate & $\begin{array}{l}\text { Las preguntas anteriores son debati- } \\
\text { das de manera colectiva sin la ayuda del } \\
\text { docente para que posteriormente vaya } \\
\text { guiando la respuesta correcta. }\end{array}$ & $15^{\prime}$ \\
\hline \multicolumn{3}{|c|}{ Sesión 4: ¿Para qué quiero separar la lluvia de la lluvia efectiva? } \\
\hline $\begin{array}{l}\text { Fase del Modelo } \\
\text { Metodológico }\end{array}$ & Descripción de la actividad & Tiempo \\
\hline Pregunta 1 & $\begin{array}{l}\text { Se les plantean dos preguntas que de- } \\
\text { ben de ser contestadas de manera indivi- } \\
\text { dual que sirven de recapitulación de las } \\
\text { tres sesiones anteriores. Son contestadas } \\
\text { y debatidas. }\end{array}$ & $10^{\prime}$ \\
\hline Teoría-Práctica & $\begin{array}{l}\text { Se van introduciendo conceptos teóricos } \\
\text { durante } 2-3 \text { minutos y los siguientes 3-4 } \\
\text { minutos se pone en práctica lo anterior. Y } \\
\text { es repetido hasta el final de la actividad. } \\
\text { Se introducen los métodos de cálculo } \\
\text { para la transformación de lluvia a caudal. }\end{array}$ & $40^{\prime}$ \\
\hline $\begin{array}{l}\text { Aplicación a la } \\
\text { realidad }\end{array}$ & $\begin{array}{l}\text { Los procesos y conceptos aprendidos du- } \\
\text { rante la anterior actividad son puestos en } \\
\text { práctica en el caso real que tiene que ser } \\
\text { entregado al final del curso. Se hace uso } \\
\text { de la herramienta ArcGis. }\end{array}$ & $40^{\prime}$ \\
\hline
\end{tabular}

Jornadas de Formación e Innovación Docente del Profesorado | № 1 (2018) Esta obra se distribuye con la licencia Creative Commons 


\begin{tabular}{|l|l|l|}
\hline Preguntas 2 & $\begin{array}{l}\text { Se plantean dos preguntas sobre el tema } \\
\text { (se les recuerda que pueden ser pregun- } \\
\text { tas de examen) para que contesten sobre } \\
\text { la marcha de manera individual. Y que } \\
\text { serán entregadas y debatidas en la si- } \\
\text { guiente sesión. }\end{array}$ & $10^{\prime}$ \\
\hline Cuestionario Final & $\begin{array}{l}\text { Se les vuelve a entregar el cuestionario } \\
\text { inicial para que contesten nuevamente } \\
\text { pero ya (supuestamente) conocidos los } \\
\text { conceptos. }\end{array}$ \\
\hline
\end{tabular}

Para evaluar el aprendizaje, hemos utilizado un Cuestionario que los estudiantes han respondido al principio y al final del CMD. Con el cuestionario Inicial (y por tanto final) se pretende que los estudiantes sean capaces de contestar unas preguntas abiertas sobre conceptos clave que deben de ser aprendidos al final de las sesiones. No son preguntas que buscan la definición correcta de los conceptos teóricos sino preguntas que les haga replantearse lo aprendido:

1. ¿Todo lo que llueve forma escorrentía? Justifica la respuesta.

2. Si la respuesta a la pregunta anterior es negativa, ¿qué variables influyen? y ¿qué necesito conocer?

3. Si anteriormente la zona de estudio ha sufrido una importante sequía, ¿cómo se ve afectado el cálculo de la precipitación que realmente forma escorrentía?

4. ¿Qué indica una abstracción inicial de 20? ¿Y de 30?

5. ¿El método racional es el único método para la transformación lluvia-caudal? Explique razonadamente. 


\section{Aplicación del ciclo de mejora}

\section{Relato resumido de las sesiones}

El material usado en todas las clases para desarrollar los conceptos teóricos y los métodos es el power point. Se trata de presentaciones amenas con mucha imagen y poco texto, para que con la visión fotográfica se queden con las ideas principales de cada una de las partes. El resumen de los aspectos más relevantes ocurridos en cada sesión se muestra a continuación.

Sesión 1: El cuestionario inicial les creó tensión al imaginar que podían ser evaluados y viendo la posibilidad de que podían dejar preguntas en blanco. Tras mucho incidir en que es un método de evaluación del docente, parecí convencerles. En la segunda parte de la sesión, el debate sobre las imágenes me llevó mas tiempo de lo previsto (inicialmente lo había planteado en 40' y acaba siendo 75'), ya que las respuestas obtenidas por los alumnos/as son muy dispares e incluso confusas. Es por esto que tengo que buscar más ejemplos que abran el debate y que me permitan introducir el tema correctamente. Tras varios ejemplos hipotéticos y reales, llegan a replantearse las ideas iniciales y ellos/as mismas llegan a la correcta resolución. La realización del esquema es algo caótico al inicio porque van diciendo ideas al tun-tun, por eso pido que digan una idea por fila. Ahora si funciona la actividad.

Sesión 2: En esta sesión lo más relevante fue la puesta en práctica de los casos, donde el alumnado empezó a dudar de lo que hacer ya que no había un enunciado fijo que seguir para la realización del problema. Paré la clase, y en la pizarra fijé los objetivos a conseguir y les pregunté que hacer para cada uno de ellos, gracias al esquema pudieron 
Llevar adelante la sesión; aunque siguen sin ser capaces de resolver los problemas sin unas directrices fijadas.

Sesión 3: En el inicio de la clase se realiza una recapitulación mediante preguntas que deben ir contestando en alto; la actividad nuevamente es caótica cuando empiezan a soltar ideas sin respetar el turno, por eso volví a fijar un turno de palabra por fila y que se pusieran de acuerdo en ello. En la segunda parte, para iniciar el siguiente tema, les pregunto cuál es la finalidad de conocer que parte de la lluvia forma escorrentía, se anotan sus ideas en la pizarra, se busca la idea en común y se va definiendo la respuesta final. Inicialmente ha sido complicado porque hay respuestas muy dispares y/o erróneas, por eso me planteo que deben de ser escritas en un lado de la pizarra y luego ir diseñando. Cuando se empieza el desarrollo de la actividad mediante las herramientas informáticas empieza a ralentizarse todo. Una vez obtenidos todos los datos, deben trabajar con ellos y sacar los gráficos que obtuvimos en la sesión anterior. Esta parte nuevamente resulta un caos, porque no ven las directrices del problema a resolver. Les digo que de manera individual planteen los objetivos y como conseguirlos, pero al final voy uno por uno corrigiendo y guiándoles.

Sesión 4: El trabajo empieza, por fin, a ver sus fruto en esta sesión. Se desarrolla toda la sesión de manera más ordenada, y respetando el turno de palabra. Un 70\% de los estudiantes empieza a ser capaz de aplicar lo aprendido en el caso real, sin necesidad de una guía. Aunque sigue habiendo un $30 \%$ que se pierde, que no sabe que hacer, incluso para a los alumnos/as que quieren seguir avanzando.

Jornadas de Formación e Innovación Docente del Profesorado | № 1 (2018) Esta obra se distribuye con la licencia Creative Commons 


\section{b) Evaluación del aprendizaje}

La evaluación del aprendizaje se ha realizado mediante las escaleras de aprendizaje (figura 3) basadas en las explicaciones desarrolladas por Porlán (2017). Estas escaleras fueron realizadas con los resultados obtenidos del cuestionario inicial (sesión 1) y del cuestionario final (sesión 4). Cada escalera es delimitada con 4 niveles, donde el nivel 0 muestra que no sabe o no conoce nada sobre los conceptos requeridos en la pregunta, y el nivel 3 que tiene todas las competencias que se requieren para desarrollar la respuesta correcta a la pregunta. Los cuestionarios fueron realizados por 18 alumnos en ambas ocasiones, y coincidió que fueron los mismos, esto me ha permitido diseñar la evaluación individual de cada alumno/a (figura 4).

En concreto aquí se van a presentar las escaleras de aprendizaje de las preguntas 2, 3 y 5 tanto del cuestionario inicial como del final, con identificación de los criterios de adecuación propuestos para cada pregunta, así como la indicación de los obstáculos a superar en cada uno de los niveles. Se puede observar como la evolución de la pregunta 2 ha sido excelente, llegando a conseguir el nivel 3 por casi el $80 \%$ del alumnado. En el caso de la pregunta 3, hay un $44 \%$ que llega a ese nivel, pero hay otro $44 \%$ que no ha sido capaz de interpretar los resultados e indicar como se harían. Y la pregunta 5 es mostrada ya que ha sido la pregunta donde no ha sido alcanzado el nivel 3 por parte de ninguno/a. Tras esta revisión quizás los principales errores que he visto son (1) posibles carencias en la explicación y (2) que las preguntas son demasiado abiertas y no se ha indicado bien lo que se pretendía encontrar. 
Pregunta 2

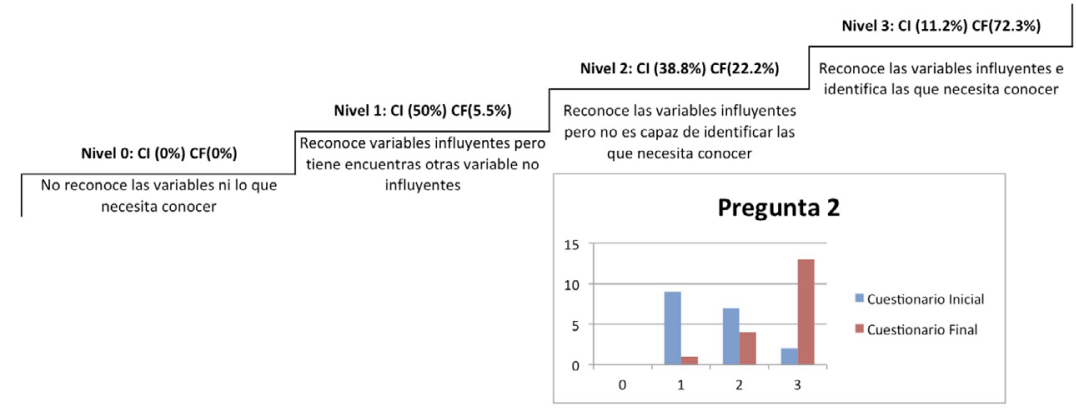

Pregunta 3

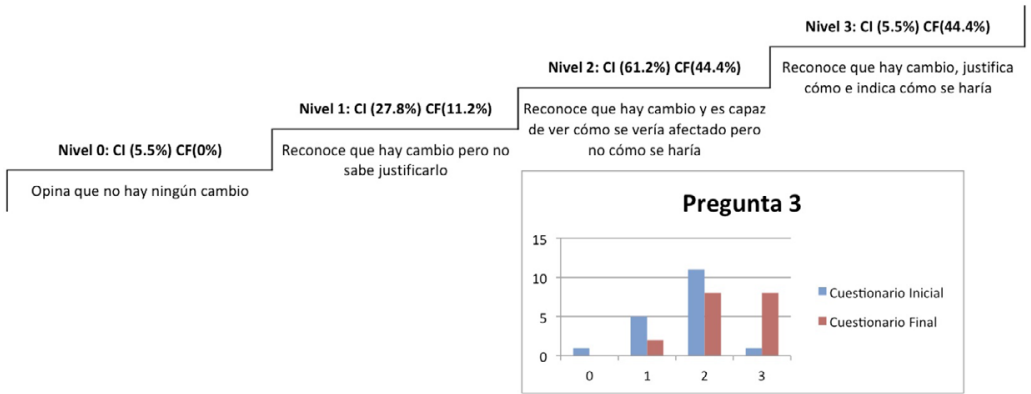

Pregunta 5

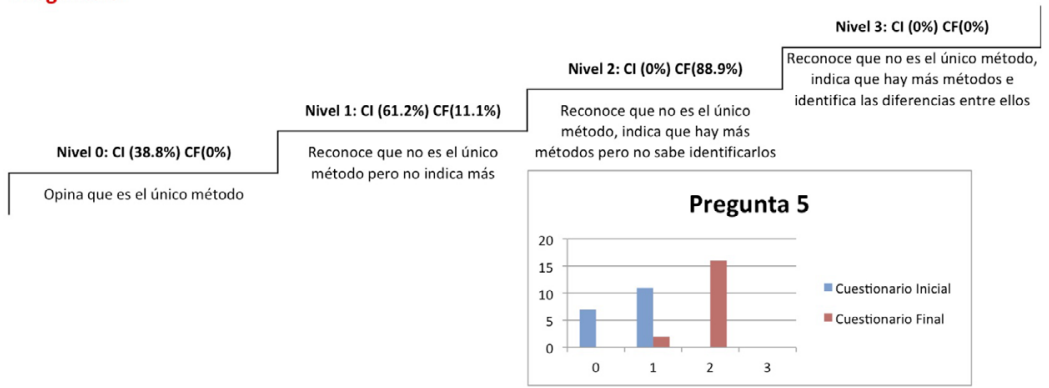

Figura 3.-Escaleras de aprendizaje de las preguntas 2, 3 y 5

De la evaluación individual (figura 4) destaca que todos los estudiantes han aprendido respecto a casi todas las cuestiones; solo en el estudiante 1 encontramos que aprende solo en dos de las preguntas. Llaman la atención dos preguntas, la 3 y 5 . En la 5 , como se ha dicho antes, se observa que ninguno/a llega al nivel 3. Y en la pregunta 3, 
que hay dos alumnos/as que bajan de nivel y que varios lo mantienen.

\begin{tabular}{|c|c|c|c|c|c|c|c|c|c|c|}
\hline \multicolumn{11}{|c|}{ Evalaación Individual del elumnado } \\
\hline \multirow{2}{*}{ Extodiame } & \multicolumn{5}{|c|}{ Cuestionario inkial } & \multicolumn{5}{|c|}{ Cuestionaris Final } \\
\hline & Pregsenta 1 & Prequita 2 & Prescantas & Prepusta 4 & Presontas 5 & Prepu-ta 1 & Pregs-nta 2 & Pregurta 3 & Presc-nta 4 & Prepu=tas \\
\hline Estudionter 1 & & & & & & & & & & \\
\hline Evtudiarte 2 & & & & & 1 & & & & & \\
\hline Estudiarte 3 & 1 & & & e & 1 & 3 & 3 & & & \\
\hline Evtudiarte 4 & 1 & & & c & tu & 3 & 5 & & & \\
\hline Estudiante 5 & 1 & & 2 & ga & 1 & 3 & 3 & 2 & & \\
\hline Entudiarte 6 & 1 & & 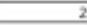 & ca & 1 & 2 & 1 & & & \\
\hline Estudiante? & 1 & & 1 & e & 1 & 3 & & & & \\
\hline Entudiarte o & 1 & & & ca & 1 & t & 3 & & & \\
\hline Estudiante 9 & & & & 0 & 0 & 5 & & & & \\
\hline Entudiarte 10 & 2 & $\frac{2}{2}$ & $\frac{2}{2}$ & בa & 1 & -2 & & & & \\
\hline Estudiante 11 & 2 & 3 & 1 & Q & ] & 3 & 3 & 2 & & \\
\hline Evtudiarta 12 & & & & ga & t & 3 & 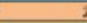 & T & & \\
\hline Estudiante 13 & & & & c & 0 & 2 & 3 & & & \\
\hline Entudiarte 14 & ] & & 3 & & 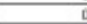 & & & & & \\
\hline Estudiante 15 & 1 & & $\underline{0}$ & 2 & 1 & & & & & \\
\hline Entudiarta 16 & 1 & & & & t & 3 & 3 & 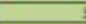 & & \\
\hline Estudiante 17 & & & & & 1 & & & & & \\
\hline Entudiarte 18 & 2 & & & ca & & & & & & \\
\hline
\end{tabular}

Figura 4.- Evaluación individual del cuestionario inicial y final (color verde suben de nivel, naranja se mantiene y rojo bajan de nivel, azul se mantienen en el máximo nivel)

\section{Evaluación del CMD}

Aunque los resultados no han sido los esperados, sí he de reconocer que la puesta en práctica de la innovación docente ha sido útil para replantear la docencia a posteriori. Creo que implica un mayor trabajo previo para su desarrollo y para acertar con las preguntas más adecuadas. Aunque la pregunta idónea saldrá a base de ensayo-error, creo que con una buena meditación con tiempo mejorarían los resultados. Considero también que el horario de clase no ha permitido desarrollar bien muchas de las actividades, ya que el Viernes a las 15:00 la participación activa por parte del alumnado era casi nula, por no decir ausente. Lo que si reconozco es que las actividades propuestas han conseguido desarrollar conceptos básicos/claves en los alumnos/as que en años anteriores era imposible, por lo abstracto del tema. La idea de plantear actividades previas ha funcionado, pero para que el papel pase por completo a ellos/as tengo que pensar y probar con otro tipo de actividades, por ejemplo trabajar en grupos y exposición al resto de la clase, en vez de que sea de manera individual y que contesten aleatoriamente. 
En el mapa de contenidos se deben relacionar más claramente los temas con preguntas abiertas, y no tanto con los apartados del tema. Por ejemplo, la parte de la transformación lluvia-caudal, debe de ser planteada mediante una imagen donde se vea una tubería y la pregunta: ¿Cómo conocer el caudal a transportar?

El modelo metodológico debería centrarse más si se puede en el alumando, donde sean ellos/as los que tomen el papel principal de las clases. Por ejemplo, se les puede plantear una pregunta, se apuntan las ideas claves (introducción del tema) en la pizarra, y luego sobre cada idea se plantean actividades a desarrollar por ellos/as y que irán contestando a cada uno de los apartados del tema. Por último, el docente pasaría a reagrupar todo y darle una estructura, con el fin de hacer la recapitulación final. El debate final se cambiaría para que sea más una actividad de cierre. Sería útil también definir el cuaderno de actividades de antemano, de manera que aunque ciertas actividades queden abiertas, sirva para delimitar los tiempos, una contra que se ha experimentado durante el ciclo.

El sistema de evaluación debería cambiar a que sólo fuesen evaluables las actividades y la participación activa de la clase, así como el trabajo final, que debe ser expuesto. Quitaría por completo la evaluación individual mediante un examen. Este método sería totalmente viable, ya que las actividades son evaluables de manera continua y se puede tener un feedback con el alumnado que permita corregirles semana a semana y les conceda la oportunidad de autocorregirse. La dificultad es el tiempo requerido por ambas partes, esto implica una dedicación profunda por parte del docente para desarrollar las actividades, corregirlas y realizar el feedback, y por parte del alumnado, que tiene que hacer más tareas y más trabajos, sabiendo que muchos de ellos/as prefieren realizar un único exámen.

Para finalizar, voy a exponer los principios didácticos que he seguido durante mi experiencia de innovación docente:

La forma de plantear la docencia para conseguir el aprendizaje del alumnado ha cambiado mediante la 
introducción de gran cantidad de actividades (de diferentes categorías: imágenes, debates, preguntas...) que involucren a los estudiantes, teniendo ellos una mayor participación y pasando el papel del docente a un segundo plano. Esta idea fue sacada de Finkel (2008) donde él guía al lector sobre como cambiar la enseñanza en base a sus principios didácticos. Uno de los más recalcados es el papel activo por parte del estudiante. La idea que busco es que el aprendizaje sea más duradero ya que ellos están "viviendo" una experiencia que puede servirles de recuerdo cuando se tengan que enfrentar a las mismas vivencias.

Para la parte del contenido, dejé a un lado la estructura jerárquica donde sólo había títulos y subtítulos y lo intercambié por preguntas que me permitan desarrollar el contenido, y es más que les permita a ellos/as mismos desarrollar el temario sin necesidad de dar una clase magistral puramente dicha. Para ello previamente se requiere de un cuaderno de actividades que te permita ir desarrollando cada uno de los aspectos definidos en el tema.

En el caso del modelo metodológico y siguiendo con la idea de los dos puntos anteriores, lo que principalmente busco es la reflexión y el debate, que sirva como una experiencia para ellos/as que les permita llevarse el aprendizaje de la clase directamente. En un futuro me gustaría añadir cada vez más participación por parte de ellos, y mantenerme como un oyente y sólo al final participar para guiarles hacia la correcta respuesta.

Por último, en cuanto a la evaluación sería útil introducirlo en todos los temas y de manera continua para que puedas ver las carencias/obstáculos que tienen el alumnado para poder reestructurar las clases y hacer un mayor hincapié en ellas. De esta manera no sólo evalúas al alumnado sino también al docente, viendo donde quizás las ideas no han sido explicadas con un suficiente detalle $y$, así, poder construir otra actividad que consiga reconducir sus ideas. 


\section{Bibliografía}

Bain, K. (2006). Lo que hacen los mejores profesores de universidad. Universidad de Valencia. Valencia, 229 págs.

Finkel, D. (2008). Dar clase con la boca cerrada. Publicaciones de la Universidad de Valencia. Valencia.

Nanía, L., Gómez, M. (2007). Ingeniería Hidrológica . Grupo Editorial Universitario Granada.

Porlán R. (2017). Enseñanza Universitaria. Cómo Mejorarla. Madrid: Morata.

Sánchez San Román, F.J., (2017). Hidrología Superficial y Subterránea. Apuntes de hidrología, Universidad de Salamanca.

Te Chow, V. (1994). Hidrología aplicada. McGraw-Hill. 